\title{
Silencing of the $N$ Family of Resistance Genes in Nicotiana edwardsonii Compromises the Hypersensitive Response to Tombusviruses
}

\author{
Boovaraghan Balaji, John Cawly, Carlos Angel, Zhanyuan Zhang, Karuppaiah Palanichelvam, \\ Anthony Cole, and James Schoelz \\ Division of Plant Sciences, University of Missouri, Columbia 65211, U.S.A.
}

Submitted 26 April 2007. Accepted 18 June 2007.

\begin{abstract}
The nontarget effects associated with silencing of the $N$ gene in Nicotiana edwardsonii, an amphidiploid species derived from $N$. glutinosa and $N$. clevelandii, have been characterized in this study. The $N$ protein confers resistance to Tobacco mosaic virus (TMV), and is representative of a family of nucleotide-binding site leucine-rich repeat proteins present in $N$. glutinosa. Previous studies have shown that silencing of the $N$ gene or of other plant genes associated with $N$-mediated defenses abolishes host resistance to TMV, and this effect can be measured through enhancements in movement or replication of TMV in the $N$-silenced plants. However, the nontarget effects of gene silencing have not been investigated thoroughly. Notably, are the functions of other resistance $(R)$ genes also affected in experiments designed to silence the $N$ gene? To investigate whether heterologous sequences could silence the $N$ gene, we selected an $R$ gene homolog from $N$. glutinosa that differed from the $N$ gene by approximately $17 \%$, created a hairpin transgene, and developed transgenic $N$. edwardsonii plants. Expression of this hairpin in the transgenic $N$. edwardsonii plants compromised the hypersensitive response to TMV, demonstrating that a single hairpin transgene could silence a block of $\boldsymbol{R}$ genes related by sequence similarity. We then investigated whether the response of $N$-silenced plants to other viruses would be altered, and found that the hypersensitive response triggered against the tombusviruses Tomato bushy stunt virus and Cymbidium ringspot virus also was compromised. This study indicates that a Tombusvirus $\boldsymbol{R}$ gene shares some homology with the $N$ gene, which could facilitate the cloning of this gene.
\end{abstract}

The central tenet of the gene-for-gene theory is that a plant resistance $(R)$ gene product recognizes a specific pathogen avirulence $(A v r)$ gene product, which sets in motion a cascade of plant defenses (Flor 1971). In many instances, the outcome of this interaction is a hypersensitive response (HR), a plant defense response that is characterized by the rapid death of a limited number of cells in the region of the invading pathogen

Corresponding author: James Schoelz; E-mail: schoelzj@missouri.edu

Current address of J. Cawly: Department of Biological Sciences, Southern Illinois University, Edwardsville 62026, U.S.A.

Current address of K. Palanichelvam: Plant Biology Department, The Samuel Roberts Noble Foundation, Ardmore, OK 73402, U.S.A.

Current address of A. Cole: Department of Biochemistry, Dakota Wesleyan University, Mitchell, SD 57301, U.S.A.
(Torres et al. 2002). Of over 200 virus $R$ genes that have been identified in crop plants, wild relatives, and model plant species such as Arabidopsis thaliana, $80 \%$ are inherited monogenically and many of these condition HR (Kang et al. 2005; Khetarpal et al. 1998). Although a large number of virus $R$ genes have been characterized genetically, only a handful have been physically cloned and their structure elucidated (Kang et al. 2005, Soosaar et al. 2005). Of the $R$ genes that have been cloned, many have turned out to encode receptors containing an N-terminal nucleotide binding site (NB) and a C-terminal leucinerich repeat (LRR) (Martin et al. 2003).

The NB-LRR proteins that target viruses can be divided into two groups, depending on whether the N-terminus has a coiled-coil (CC) domain or a Toll-interleukin-1 (TIR) domain. The NB-LRR R proteins that have a CC domain include Rx1, Rx2, Sw5, RCY1, HRT, and Tm2 ${ }^{2}$ (Kang et al. 2005; Martin et al. 2003; Soosaar et al. 2005). NB-LRR proteins with a TIR domain that target viruses include N (Whitham et al. 1994) and RT4-4 (Seo et al. 2006). The RT4-4 gene was isolated from common bean and targets Cucumber mosaic virus (CMV). Transgenic Nicotiana benthamiana plants that express the RT4-4 protein respond to CMV infection with systemic necrosis; thus, although this transgene does not confer complete CMV resistance in $N$. benthamiana, it is involved in recognition of CMV and activation of plant defenses. The $N$ gene is an $R$ gene derived from $N$. glutinosa whose protein product triggers HR against Tobacco mosaic virus (TMV) infections (Whitham et al. 1994). The $N$ gene was the first virus $R$ gene to be cloned and has been the object of intense study.

Gene silencing is one key technique that has emerged in recent years for investigating the function of $R$ genes, including the $N$ gene. Plant genes can be silenced in one of two ways, either through the expression of hairpin transgenes or through virus expression vectors (i.e., virus-induced gene silencing [VIGS]) (Baulcombe 1999; Matzke et al. 2001; Robertson 2004; Smith et al. 2000). VIGS has been used to target the $N$ gene for silencing, as well as several other key plant genes involved in $N$-mediated defenses. Silencing of the $N$ gene or of other genes involved in $N$-mediated defenses will abolish host resistance to TMV, and this effect can be measured through enhancements in movement or replication of TMV in the $N$-silenced plants (Jin et al. 2002; Liu et al. 2002, 2004; Lu et al. 2003; Peart et al. 2002, 2005). However, the nontarget effects of gene silencing have not been investigated thoroughly. Notably, are the functions of other $R$ genes also affected in experiments designed to silence the $N$ gene?

In this study, we have characterized the nontarget effects associated with silencing of the $N$ gene in $N$. edwardsonii, an 
amphidiploid species derived from $N$. glutinosa and $N$. clevelandii (Christie 1969). We recently proposed that $N$. edwardsonii could serve as a reservoir for a broad range of virus $R$ genes, including a new $R$ gene that targets at least five distinct tombusviruses (Schoelz et al. 2006). We now show that a single hairpin transgene could be used to silence a block of plant $R$ genes related by sequence identity to the $N$ gene, and that the tombusvirus resistance in $N$-silenced plants is compromised. This study indicates that a tombusvirus $R$ gene may be a member of the $N$ family of $R$ genes.

\section{RESULTS}

\section{Characterization of the $N$ family of $\boldsymbol{R}$ gene homologs in Nicotiana spp.}

A nucleotide sequence analysis of all TIR-NB-LRR $R$ genes has revealed several conserved amino acid stretches present in exon 2, including the motifs GGVGKTT and GLPLAL. Oligonucleotide primers based on these motifs can be used in polymerase chain reaction (PCR) to amplify portions of true $R$ genes and related $R$ gene homologs (RGHs) from plant genomes (Kanazin et al. 1996; Leister et al. 1996; Yu et al. 1996). To assess the diversity of $N$-related sequences present in $N$. glutinosa and $N$. clevelandii, we chose forward and reverse oligonucleotide primer sequences directed against the amino acid motifs GGVGKTT and GLPLAL, respectively, which precisely matched the $N$ gene sequence. These primers yielded RGH clones that varied in size from 498 to $516 \mathrm{bp}$ and whose nucleotide sequences differed from the $N$ gene by 0 to $17 \%$.

We obtained 77 RGH clones derived from $N$. glutinosa, and 45 of these sequences contained complete open reading frames (ORFs) with a size of $516 \mathrm{bp}$. The remaining 32 clones contained deletions or point mutations that altered the reading frame of exon 2 in a manner that led to the introduction of stop codons; these clones were considered to be pseudogenes and were excluded from further analysis. The 45 clones that had complete ORFs could be aligned into 11 groups that varied in identity to the $N$ gene from 87 to $100 \%$ (Fig. 1). To account for possible PCR error, nucleotide sequences that differed by $\leq 1 \%$ were grouped together as a single sequence, in agreement with previous studies (Couch et al. 2006; Plocik et al. 2004). Consequently, we propose that $N$. glutinosa contains at least 10 RGHs that are closely related in sequence to the true $N$ gene; they constitute the $N$ family of RGHs. This number is in agreement with a previous estimate of 8 to $10 \mathrm{~N}$-related RGHs in Nicotiana spp. (Whitham et al. 1994). Although the PCR primers precisely matched the $N$ gene sequence, the $N$ gene itself was represented in only one-fifth of the RGH sequences, as this group contained only eight members derived from N. glutinosa (Fig. 1). Interestingly, RGH sequences that were described previously as $N$ homologs, such as NH, Y-1, NL-25, and NL-27 (Hehl et al. 1999; Stange et al. 2004; Vidal et al. 2002), were not associated with any of the 11 groups derived from $N$. glutinosa (Fig. 1).

We sequenced 30 RGH clones derived from $N$. clevelandii, and 28 of these sequences contained complete ORFs with a size of $516 \mathrm{bp}$. These RGHs were a subset of those isolated from $N$. glutinosa, because all 28 fell within one of four previously identified $N$. glutinosa RGH groups (Fig. 1). Interestingly, the $N$ gene group was one of the four that contained $N$. clevelandii RGHs. Four of the $N$. clevelandii RGH sequences were identical to the $N$ gene over their 516-bp length, and another seven differed from the $N$ gene by less than $1 \%$. Although $N$. clevelandii is susceptible to TMV, the isolation of an RGH group that matched the $N$ gene sequence indicated that this host might contain a form of the $N$ gene inactivated through a point mutation at another location or through a premature termina- tion of the downstream coding region. Because $N$. glutinosa and $N$. clevelandii are the parent species of $N$. edwardsonii, this analysis showed that $N$. edwardsonii contained multiple $N$ homologs that would be affected by a silencing strategy that targeted the true $N$ gene.

\section{Silencing of the $N$ gene with hairpin transgene sequences that are identical to the $N$ gene.}

To investigate whether a hairpin transgene could silence a block of $R$ genes related to each other by sequence similarity, we first developed a set of transgenic control plants that expressed a hairpin transgene that was based on exon 1 of the $N$ gene (Fig. 2). The mRNA expressed from the exon 1 transgene would be expected to fold into a hairpin that had a length of 239 nucleotides. We regenerated 30 putative transformants and we verified that 23 of these $\mathrm{T}_{0}$ plants expressed NPTII. The plants positive for NPTII were subsequently evaluated for their response to $\mathrm{TMV}$, and we found that $56 \%$ (13 of $23 \mathrm{~T}_{0}$ lines) exhibited silencing of the $N$ gene in a detached leaf assay (Table 1). Wild-type N. edwardsonii detached leaves consistently developed HR by 2 days post inoculation (dpi). In contrast, detached leaves from plants that exhibited silencing of the $N$ gene exhibited no sign of HR at 2 dpi. Eventually, the $N$-silenced plants did develop necrotic lesions, but HR development was delayed at a minimum to $3 \mathrm{dpi}$ and, in some lines, HR did not appear until 6 dpi, 4 days after the appearance of HR in wild-type $N$. edwardsonii.

To confirm that the $N$-silenced plants could support the replication of TMV at early time points before the onset of HR, we utilized TMV-30B-green fluorescent protein (GFP) (Shivprasad et al. 1999). The response of wild-type and $\mathrm{N}$-silenced transgenic $N$. edwardsonii to TMV-30B-GFP at $5 \mathrm{dpi}$, visualized under visible and UV light, is illustrated in Figure 3. The $N$-silenced plants responded with a delayed HR (compare Fig. 3A with B), but GFP expression was much higher in the $N$-silenced plants (compare Fig. 3E with F) than in the wildtype plants. In fact, no GFP expression was associated with lesions in wild-type $N$. edwardsonii at 5 dpi. These experimental results are in agreement with previous studies in which the $N$ gene was silenced with a VIGS vector (Jin et al. 2002; Liu et al. 2002; Peart et al. 2002). Interestingly, a comparison of TMV titers in $N$-silenced versus wild-type $N$. edwardsonii leaves by either enzyme-linked immunosorbent assay (ELISA) or reversetranscriptase (RT)-PCR did not reveal any consistent differences (data not shown), indicating that the spread of TMV infections was not limited by HR within this timeframe.

To determine whether the $N$-silencing phenotype would be carried into subsequent generations, two $\mathrm{T}_{0}$ lines were selfed twice and evaluated for their response to TMV inoculation. Plants in the $T_{1}$ generation exhibited a delay in HR up to 4 days, because HR did not develop until 6 dpi. Plants in the $T_{2}$ generation exhibited delays in HR up to 1 day, because HR in these plants did not develop until 3 dpi (data not shown). Some of the progeny in the $T_{1}$ or $T_{2}$ generations did not respond to TMV with any delay in HR, indicating that these plants might have undergone transcriptional silencing of the hairpin transgene.

\section{Expression of $N$ gene transcript \\ in putatively $N$-silenced $N$. edwardsonii.}

To further show that the delayed HR phenotype observed in putatively $N$-silenced $N$. edwardsonii was due to reductions in the $N$ transcript level, real-time PCR was used to quantify the transcript of the $N$ gene in wild-type $N$. edwardsonii and in putatively $N$-silenced $N$. edwardsonii transgenic lines. To assess the level of $N$ transcript, PCR primers were synthesized that would amplify a 140-bp DNA segment of exon 4, the 
same primers used by Levy and associates (2004) in their study of $N$ transcript levels in TMV-infected and uninfected plants. Quantification of the $N$ gene transcript level was determined by comparing the ratios of $N$ gene transcript with EF$1 \alpha$ transcript in wild-type and $N$-silenced $N$. edwardsonii. A dissociation curve completed at the end of the PCR reactions demonstrated that each of the primer pairs tested for the $N$ gene and for EF- $1 \alpha$ amplified a single PCR product with a distinct melting temperature $(\mathrm{Tm})$ as indicated by the fluorescence $(-\mathrm{dF} / \mathrm{dT})$ against the temperature.

Two $\mathrm{T}_{1}$ seedlings from transgenic line $\mathrm{K} 242$ (K242-A5 and K242-A8) and four $T_{2}$ seedlings derived from K242-A9 (K242-A9-10, K242-A9-11 K242-A9-1, and K242-A9-15) were selected for the analysis of $N$ gene transcript level, as

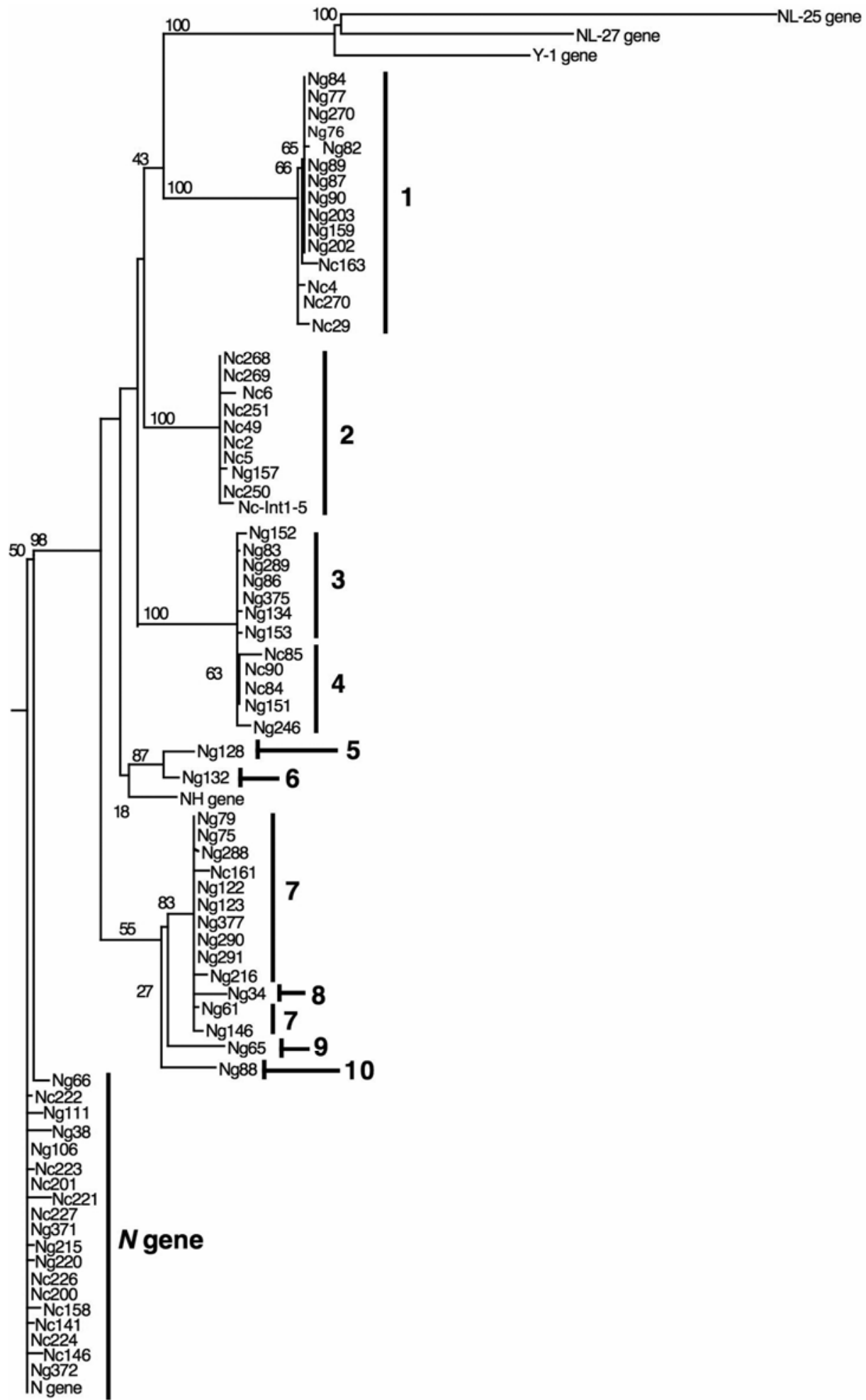

Fig. 1. Phylogenetic analysis of Nicotiana glutinosa $(\mathrm{Ng})$ and $N$. clevelandii $(\mathrm{Nc}) R$ gene homologs (RGHs). The tree is based on the alignment of nucleotide sequences of RGH clones, the $N$ gene, and four published $N$ homologs $(N H, N L-25, N L-27$, and $Y-1)$. Bootstrapping values on the branches are shown as a percentage based on 1,000 replicas. To account for possible polymerase chain reaction error, nucleotide sequences that differed by $\leq 1 \%$ were considered to be representative of a single sequence. 
well as leaves from wild-type $N$. edwardsonii. Individual leaves were divided into two samples along the midrib. One half-leaf was inoculated with TMV to verify the delayed-HR phenotype, whereas total RNA was isolated from the other half-leaf for real-time PCR analysis of the $N$ transcript. Our results show that, when the level of $N$ gene was normalized to the value of 1 for wild-type $N$. edwardsonii, the level of $N$ gene transcript in transgenic $N$. edwardsonii leaves that exhibited a delayed HR was significantly reduced (Fig. 4). Significantly, the $\mathrm{T}_{2}$ line K242-A9-1 did not exhibit a delayed HR in response to TMV inoculation, and the level of $N$ gene transcript in this line was equivalent to that of the wild-type $N$. edwardsonii. This experiment showed that the delayed HR phenotype in transgenic plants that expressed an $N$ gene hairpin was associated with a reduction in the level of the $N$ transcript.

\section{Silencing of the $N$ gene by a hairpin transgene developed from a heterologous sequence.}

To investigate whether a hairpin transgene derived from a heterologous sequence could silence the $N$ gene, we selected an RGH that differed from the $N$ gene by approximately $17 \%$ within the sequence of the hairpin (Fig 5). The mRNA expressed from the RGH3 transgene (Fig. 2) would be expected to fold into a hairpin that had a length of 120 nucleotides, including a stretch of 26 nucleotides that was identical to the published $N$ gene sequence, corresponding to nucleotides 1,423 to 1,448 (Fig. 5). We regenerated 24 putative transformants and verified that 13 of these $T_{0}$ plants expressed NPTII. The plants positive for NPTII subsequently were evaluated for their response to TMV, and we found that $62 \%$ ( 8 of $13 \mathrm{~T}_{0}$ lines) exhibited silencing of the $N$ gene in our detached leaf assay (Table 1). As with the $N$ gene hairpin, eventually the leaves from the RGH3 transgenic plants did develop HR, but HR development was delayed at a minimum to 3 dpi and, in some lines, did not appear until 5 dpi, 3 days after its appearance in wild-type $N$. edwardsonii. The experiments with the RGH3 hairpin showed that heterologous $R$ gene sequences could silence a true $R$ gene and, consequently, that a single hairpin transgene could silence a block of $R$ genes related by sequence similarity.

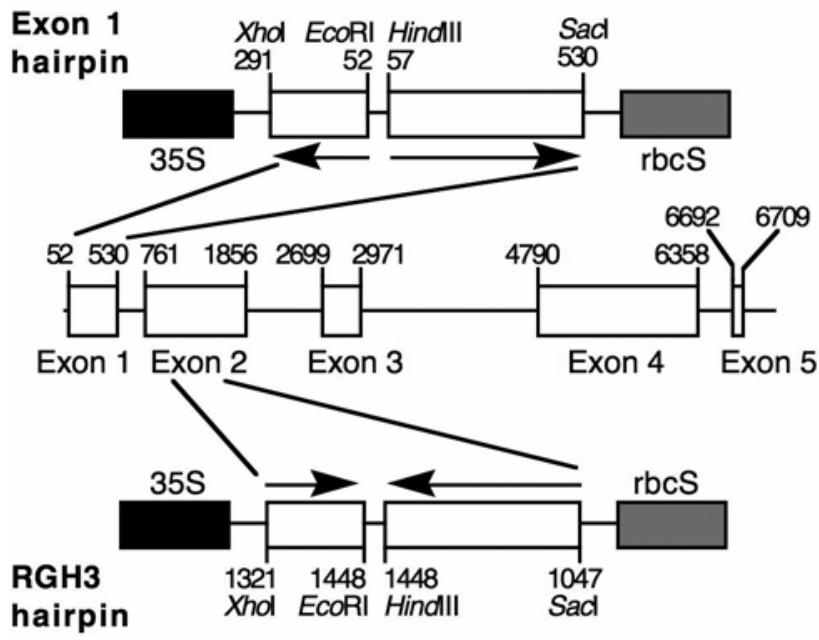

Fig. 2. Composition of hairpin constructs used for silencing of the $N$ gene in Nicotiana edwardsonii. The structure of the $N$ gene, with its five exons, is illustrated in the middle of the figure. The promoter and terminator for the hairpin transgenes are illustrated by filled and gray boxes, respectively. The Exon 1 hairpin is derived from the $N$ gene sequence, whereas the $R$ gene homolog (RGH3) hairpin is derived from the RGH3 sequence.

\section{Silencing of the $N$ family of $R$ genes compromises} resistance to two tombusviruses.

Our gene-silencing assay demonstrated that resistance to TMV could be silenced effectively by a homologous exon 1 hairpin transgene or a heterologous exon 2 hairpin transgene. However, the true utility of our silencing assay would be to show that silencing of some or all members of the $N$ family of $R$ genes would abolish resistance to a virus unrelated to TMV. To investigate whether the response of $N$-silenced plants to other viruses would be altered, we inoculated two tombusviruses, Tomato bushy stunt virus (TBSV) and Cymbidium ringspot virus (CymRSV), to wild-type $N$. edwardsonii and transgenic $N$. edwardsonii that expressed the exon 1 hairpin. We selected the tombusviruses for the detached leaf assay because $N$. edwardsonii responds with a dominant HR (Chu et al. 1999; Schoelz et al. 2006) and these viruses are very aggressive, eliciting HR within 2 dpi. TMV was inoculated to a separate set of detached leaves from the same plants to verify silencing of the $N$ gene. In the detached-leaf assay, both TBSV and CymRSV induced HR in wild-type $N$. edwardsonii by 2 dpi (Fig. 3C and D), whereas no individual lesions were induced by either virus in the $N$-silenced plants at the same time point (Fig. $3 \mathrm{G}$ and $\mathrm{H}$ ). A comparison of TBSV titers in $N$-silenced versus wild-type $N$. edwardsonii leaves by ELISA did not reveal any consistent differences (data not shown), indicating that spread of TBSV infections were not limited by HR within this time frame. Furthermore, the silencing of the TBSV HR was transitory, because detached leaves eventually developed HR by 5 dpi. These results were comparable with what was found in the assessment of silencing of the $N$ gene. Given that TBSV resistance segregates independently from the $N$ gene (Schoelz et al. 2006), these silencing results indicate that the tombusvirus $R$ genes belong to the $N$ family of $R$ genes, but are distinct from the $N$ gene.

\section{DISCUSSION}

In this article, we showed that strategies designed to target the $N$ gene for post-transcriptional gene silencing also target a family of $N$ gene homologs present in Nicotiana spp. As a prerequisite to the silencing study, we used a PCR-based approach to estimate the number of $N$ gene homologs in Nicotiana spp. that might be affected by silencing of the $N$ gene. We found that $N$. glutinosa contained at least 10 homologs in addition to the $N$ gene, whereas $N$. clevelandii contained at least 4 homologs. Interestingly, the $N$ gene homologs found in $N$. clevelandii were a subset of those present in N. glutinosa. Because $N$. edwardsonii is an amphidiploid composed of $N$. glutinosa and $N$. clevelandii parents (Christie 1969), it must contain at least $14 N$ gene homologs in addition to the true $N$ gene.

To create transgenic $N$. edwardsonii plants that were $N$-silenced, we developed two hairpin constructs that targeted exon 1 and exon 2 of the $N$ gene. Exon 2 of the $N$ gene was targeted for silencing by an RGH sequence (RGH3) that differed from the published $N$ sequence (Whitham et al. 1994) by approxi-

Table 1. Response of transgenic plants expressing the Exon 1 or $R$ gene homolog (RGH3) hairpin transgene

\begin{tabular}{lcc}
\hline Characteristic & Exon 1 & RGH3 \\
\hline Length of hairpin $^{\mathrm{a}}$ & $236 \mathrm{nt}$ & $120 \mathrm{nt}$ \\
Homology with $N$ gene $_{\text {No. of primary transformants }}$ & Identical & $83 \%$ \\
$\mathrm{~T}_{0}$ plants exhibiting silencing of the $N$ gene & $57 \%(13 / 23)$ & $62 \%(8 / 13)$ \\
\hline
\end{tabular}

${ }^{\text {a }}$ Length in nucleotides (nt). 
mately $17 \%$, demonstrating that more than one $\mathrm{R}$ gene could be effectively silenced by a single hairpin, as long as sufficient sequence homology exists between the hairpin sequence and the $R$ gene targets. In particular, there is a short stretch of 26 nucleotides (from nucleotide 1,423 to 1,448 ) in the hairpin construct based on RGH3 that has $100 \%$ homology to the $N$ gene sequence. Other RGH3 sequences also might have participated in silencing of the $N$ gene, but this stretch of $26 \mathrm{nu}$ cleotides is sufficient to induce silencing of the $N$ gene in plants. Klahre and associates (2002) have shown that a sequence containing as few as 21 nucleotides of dsRNA can efficiently silence a target gene.

We utilized real-time PCR to confirm that the $N$ gene was silenced, using primers derived from exon 4 of the $N$ gene, downstream of the sequences that were targeted by our hairpin transgenes. We chose this target because it had been used successfully by others to measure $N$ transcript levels (Levy et al. 2004). The exon 4 sequence is not spliced out during alternative splicing of the $N$ gene (Dinesh-Kumar and Baker 2000); therefore, the levels of both the full-length and truncated, alternatively spliced transcripts would be assessed. In the real-time PCR assay, we observed that our hairpin transgene caused a reduction of $N$ gene transcripts by 60 to $80 \%$ compared with wild-type $N$. edwardsonii. This was comparable to the reductions observed in the $N$ gene mediated by VIGS (Liu et al. 2002). In a related bioassay with TMV inoculations, these leaves exhibited a 1- to 5-day delay in the HR response relative to wild-type $N$. edwardsonii leaves. The inability to abolish the $N$ gene transcripts might explain why we observed a delay in the development of HR rather than a complete abolishment. Another intriguing possibility may be that, as the virus replicates, its silencing suppressor might interfere with the induction of RNAi by the hairpin mRNA, which would lead to a restoration in the function of the $R$ genes.

Once we confirmed that the $N$ gene in $N$. edwardsonii had been silenced, we then investigated whether the response of $N$ silenced plants to viruses other than TMV would be altered. We found that the HR triggered against the tombusviruses TBSV and CymRSV also was compromised. Our results, therefore, suggest that the nucleotide sequence of the tombusvirus $R$ gene must have a high degree of homology to the $N$ gene. Although the tombusvirus and TMV $R$ genes are both derived from $N$. glutinosa, they segregate independently (Cole et al. 2001; Schoelz et al. 2006), which demonstrates that the $N$ gene itself does not specify resistance to either TBSV or CymRSV. Consequently, $R$ genes closely related by sequence to the $N$ gene might be considered candidates for the new tombusvirus $R$ gene. Although resistance to TBSV and CymRSV segregate together (Schoelz et al. 2006), we do not know yet if a single gene specifies resistance to both viruses or if separate $R$ genes target each virus individually. However, our observation that silencing of the $N$ gene affects resistance to both TBSV and CymRSV indicates that their $R$ genes are related at least at the sequence level, and this information should facilitate the cloning of these new $R$ genes.

Three strategies have been employed to identify and clone virus $R$ genes. The $N$ and $T m 2^{2}$ genes were cloned by transposon tagging (Lanfermeijer et al. 2003; Whitham et al. 1994), whereas the recessive potyvirus $R$ genes were cloned through a candidate gene approach focused on $e I F 4 E$ (Nicaise et al. 2003; Ruffel et al. 2002). The most common strategy for the physical isolation of virus $R$ genes has involved the
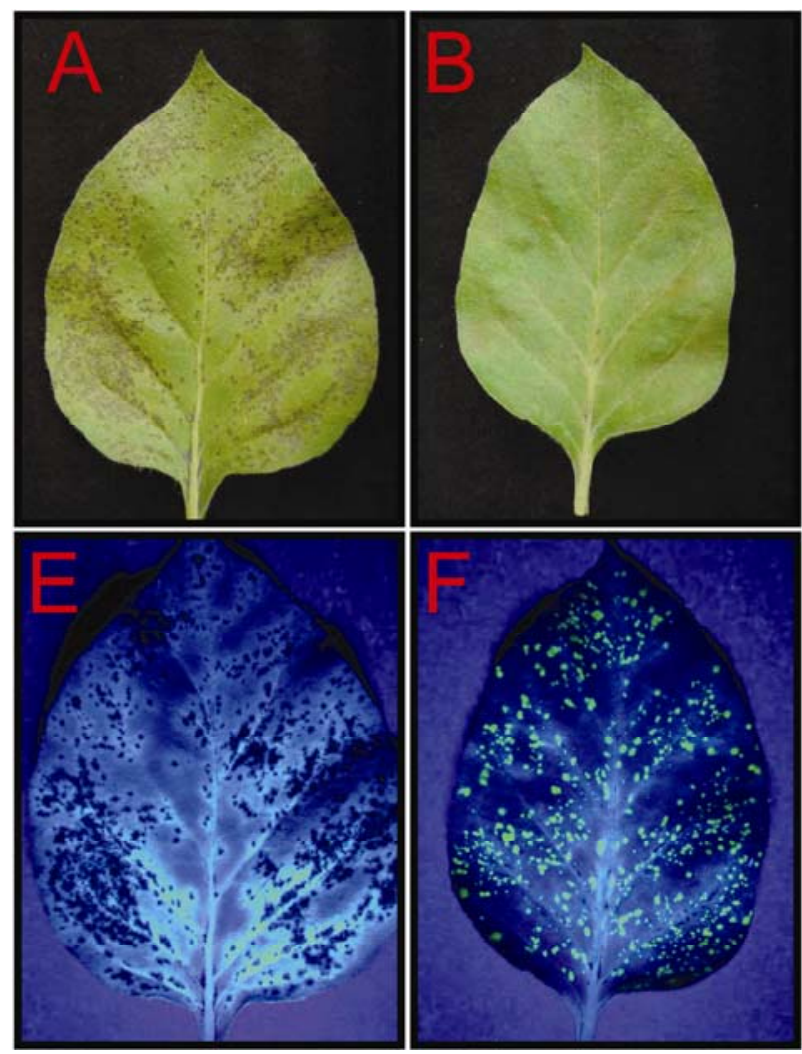
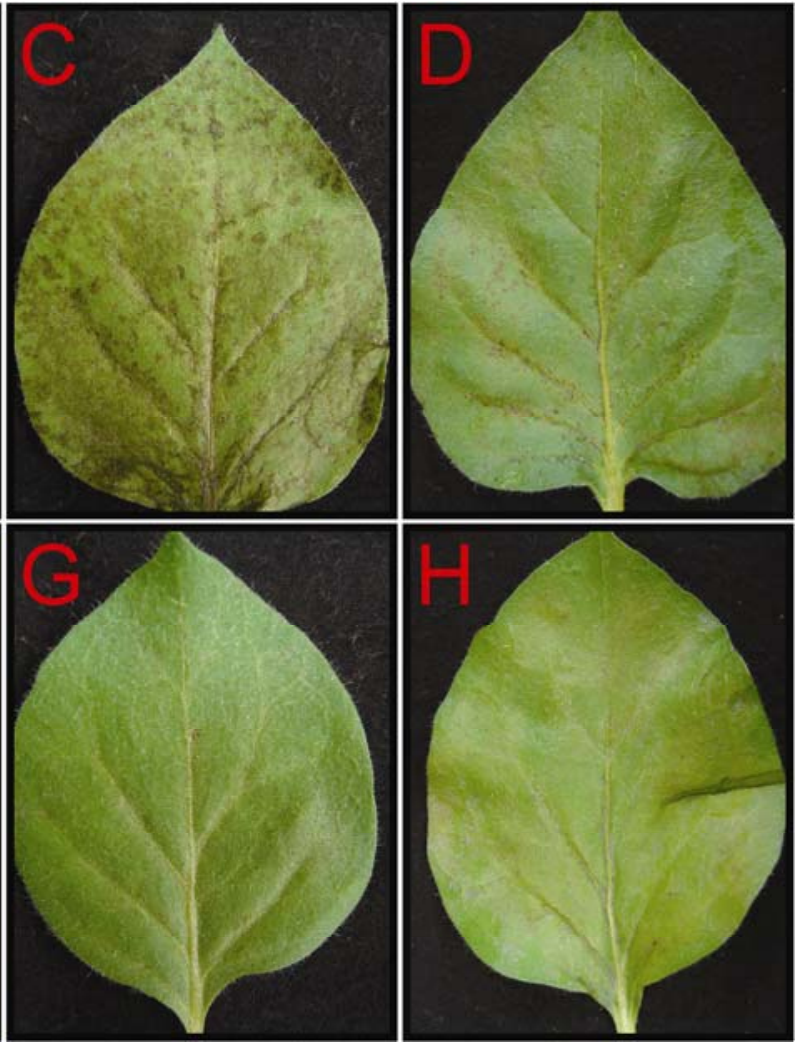

Fig. 3. Response of wild-type and $N$-silenced transgenic Nicotiana edwardsonii to inoculation with Tobacco mosaic virus (TMV)-30B-green fluorescent protein (GFP), Tomato bushy stunt virus (TBSV), or Cymbidium ringspot virus (CymRSV). A, Wild-type $N$. edwardsonii leaf inoculated with TMV-30B-GFP, at 6 days post inoculation (dpi). B, $N$-silenced transgenic $N$. edwardsonii leaf inoculated with TMV-30B-GFP, at 6 dpi. C, Wild-type $N$. edwardsonii leaf inoculated with TBSV, at 4 dpi. D, Wild-type $N$. edwardsonii leaf inoculated with CymRSV, at 4 dpi. E, Same leaf as in panel A, under UV light. F, Same leaf as in panel B, under UV light. G, $N$-silenced transgenic $N$. edwardsonii leaf inoculated with TBSV, at 4 dpi. H, $N$-silenced transgenic $N$. edwardsonii leaf inoculated with CymRSV, at 4 dpi. 
use of extensive physical maps and positional cloning (Kang et al. 2005). Each of the techniques used for cloning $R$ genes has its strengths and weaknesses. For example, transposon tagging of the $N$ gene was successful in part because TMV has a very stable virion that is highly infectious. Consequently, large numbers of plants could be spray inoculated to find the rare mutant that contained an $N$ gene disrupted by the transposon (Whitham et al. 1994). Similarly, the candidate gene approach has worked well for isolation of recessive potyvirus $R$ genes, but this requires some knowledge of the identity of the $R$ gene before it can be cloned. Thus far, positional cloning has been a reliable technique that has been used for the identification of most $R$ genes, regardless of the type of pathogen targeted (Martin et al. 2003). However, positional cloning works best in plants that have good genetic and physical maps, something that does not exist for every plant species. Furthermore, some $R$ genes might be located in genomic regions where crossovers are rarely observed.

Our demonstration that gene silencing technology can be effective in identifying a previously unrecognized tombusvirus $R$ gene has the potential to augment existing techniques for characterization and cloning new $R$ genes. In particular, it could facilitate the cloning of valuable disease $R$ genes in plants such as $N$. edwardsonii, a species that could serve as a reservoir for virus $R$ genes (Schoelz et al. 2006) but for which no physical map exists. It is impractical to isolate genomic clones of $R$ genes and randomly test them individually for their reaction to a specific pathogen; nevertheless, it is possible to find new $R$ gene specificities using a gene-silencing assay that shuts off entire blocks of related $R$ genes. This assay could narrow the search to a manageable number of $R$ gene candidates that could be identified through Southern hybridization of an N. glutinosa genomic library. Previous work has shown that the TBSV P22 protein is responsible for elicitation of HR in N. edwardsonii (Chu et al. 1999). Consequently, individual $R$ genes could be tested for HR activity through co-agroinfil-

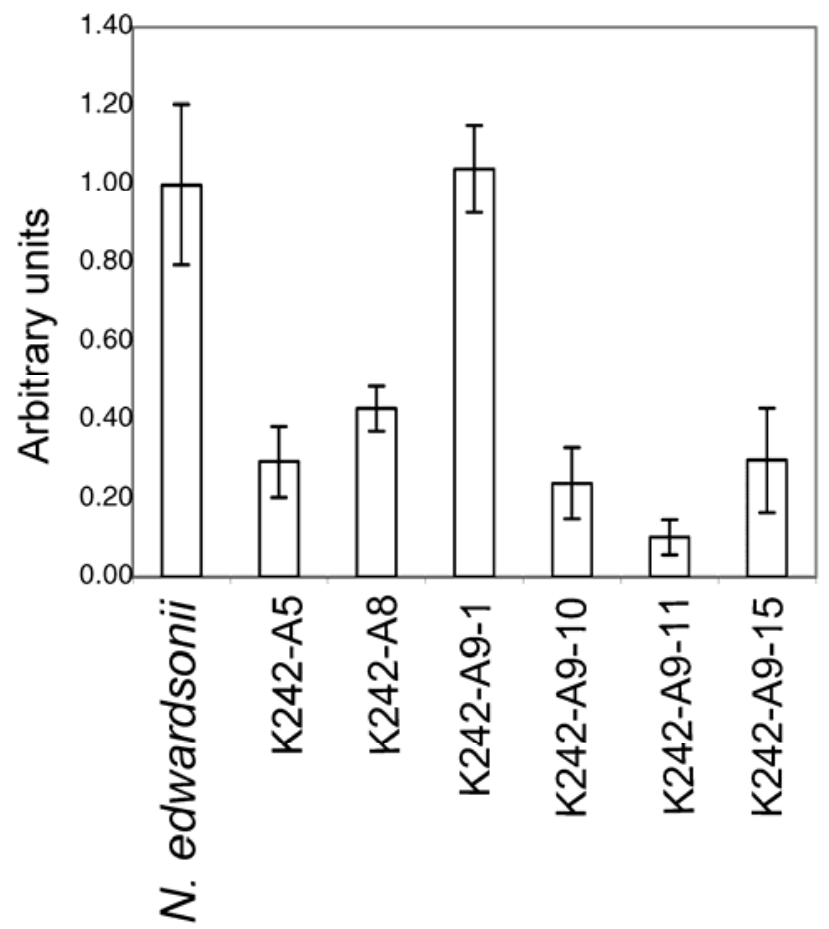

Fig. 4. Assessment of $N$ gene transcript levels in wild-type Nicotiana edwardsonii and $N$-silenced transgenic $N$. edwardsonii by real-time polymerase chain reaction. tration with the TBSV p22 gene into a susceptible solanaceous host such as $N$. clevelandii.

Although we have shown that silencing of the $N$ family of $R$ genes compromises the HR to TBSV, one alternative hypothesis would lead to the conclusion that the TBSV $R$ gene might be unrelated by sequence to the $N$ gene. Peart and co-workers (2005) recently found that silencing of the gene NRG1 in $N$. edwardsonii abolished resistance to TMV, yet $N R G 1$ encoded a CC-NB-LRR gene that did not have sequence homology to $N$. The implication was that disease resistance pathways may recruit more than a single NB-LRR protein and, in fact, effective resistance may require multiple NB-LRR proteins. Similarly, we have found that silencing of the $N$ family of $R$ genes compromises the resistance response of $N$. edwardsonii to TBSV. Consequently, there is a possibility that the $\mathrm{N}$ family of proteins may participate in TBSV-mediated resistance, even though the TBSV $\mathrm{R}$ protein is unrelated to this family. A more complete characterization of the members of the $N$ family of $R$ genes should reveal whether the TBSV $R$ gene belongs to this group.

\section{MATERIALS AND METHODS}

Isolation of genomic DNA and construction of RGH clones.

Genomic DNA was isolated from Nicotiana spp. according to Dellaporta and associates (1983). Primer sequences for PCR amplification of the $N$ gene and related $\mathrm{RGH}$ sequences were derived from the published $N$ gene sequence (Whitham et al. 1994) (GenBank accession U15605). To amplify and clone RGH sequences, the forward primer corresponded to nucleotides 933 to 950 of the $N$ gene and an EcoRI site was included on the $5^{\prime}$ end of the primer to facilitate cloning (5'-GGGGAA TTCGGGGGAGTCGGTAAAACACA-3'). The reverse primer corresponded to nucleotides 1,431 to 1,448 of the $N$ gene and a $K p n I$ site was included on the $5^{\prime}$ end of the primer to facilitate

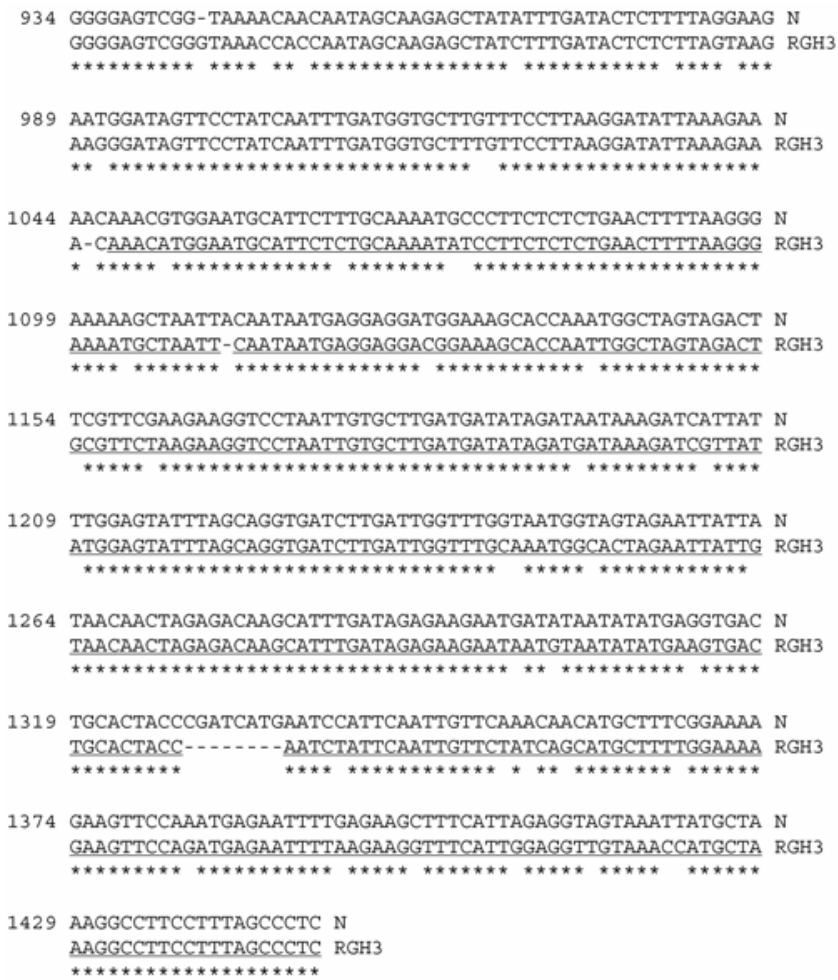

1264 TAACAACTAGAGACAAGCATTTGATAGAGAAGAATGATATAATATATGAGGTGAC N TAACAACTAGAGACAAGCATTTGATAGAGAAGAATAATGTAATATATGAAGTGAC RGH3

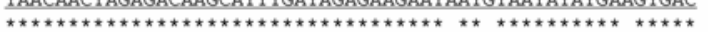

1319 TGCACTACCCGATCATGAATCCATTCAATTGTTCAAACAACATGCTTTCGGAAAA N

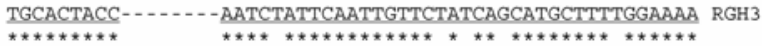

1374 GAAGTTCCAAATGAGAATTTTGAGAAGCTTTCATTAGAGGTAGTAAATTATGCTA N GAAGTTCCAGATGAGAATTTTAAGAAGGTTTCATTGGAGGTTGTAAACCATGCTA RGH3

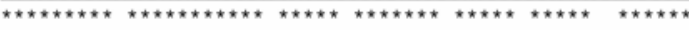

1429 AAGGCCTTCCTTTAGCCCTC N AAGGCCTTCCTTTAGCCCTC RGH

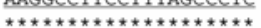

Fig. 5. Comparison of $R$ gene homolog (RGH3) nucleotide sequence to published $N$ gene sequence. The portion of the RGH3 nucleotide sequence was used for the development of the RGH3 hairpin transgene is underlined. 
cloning (5'-GGGGGTACCGAGGGCTAAAGGAAGGCC-3'). PCR conditions were $94^{\circ} \mathrm{C}$ for $5 \mathrm{~min}, 55^{\circ} \mathrm{C}$ for $30 \mathrm{~s}$, and $72^{\circ} \mathrm{C}$ for $2 \mathrm{~min}$ for 1 cycle, followed by 35 cycles at $94^{\circ} \mathrm{C}$ for $30 \mathrm{~s}$, $55^{\circ} \mathrm{C}$ for $30 \mathrm{~s}$, and $72^{\circ} \mathrm{C}$ for $90 \mathrm{~s}$. The amplified DNA subsequently was cloned into the EcoRI-KpnI restriction enzyme sites of pGem-7Zf(+) or into pGEM-T easy (Promega Corp., Madison, WI, U.S.A.) to create the RGH clones. RGH nucleotide sequences were determined at the Nucleotide Sequencing Core Facility at the University of Missouri.

\section{Analysis of RGH nucleotide sequences.}

Sequences were manually edited, cleaned of vector and primer sequences, and initially compared using BLAST-N (Altschul et al. 1990). All values were set to default and the low-complexity filter was used. Nucleotide sequence alignments were performed using ClustalX1.83 (Thompson et al. 1997) with phylip output format and edited in Jalview (Clamp et al. 2004). Parsimony trees were calculated using the program "protpars" of Phylip (Felsenstein 2000). Bootstrap values were calculated using the program "seqboot" of Phylip package followed by "protpars" on 1,000 individual data sets. Trees were viewed and rooted using ATV (Zmasek and Eddy 2001).

Sequence errors in the RGHs due to Taq polymerase were estimated to be equal to or less than $1 \%$, in agreement with previous studies (Couch et al. 2006; Plocik et al. 2004).

\section{Construction of $\boldsymbol{R}$ gene hairpins \\ for transformation of $N$. edwardsonii.}

Two hairpin constructs were evaluated for their capacity to silence the $N$ gene. One sequence was derived from exon 1 of the $N$ gene, whereas the RGH3 hairpin was derived from an exon 2 sequence isolated from $N$. edwardsonii (Fig. 2). To construct the exon 1 hairpin, $N$ gene sequences from nucleotide 55 to 291 were amplified by PCR from N. edwardsonii genomic DNA using the oligonucleotide forward primer $5^{\prime}$ GGGGAATTCGCATCTTCTTCTTCTTCTTCT-3' and reverse primer 5'-GGGCTCGAGTGATGTTGCATAATTCTCTGA-3' The forward primer included an EcoRI site on the 5' end, whereas the reverse primer had an XhoI site on its $5^{\prime}$ end. PCR conditions were the same as for the RGH clones. The amplified DNA subsequently was cloned into the EcoRIXhoI restriction enzyme sites of $\mathrm{pGem}-7 \mathrm{Zf}(+)$ and the clone was designated pNE1. To complete the hairpin, $N$ gene sequences from 55 to 531 were amplified by PCR from $N$. edwardsonii genomic DNA using the forward primer 5'-GG GAAGCTTGCATCTTCTTCTCTTCTTCT-3' and the reverse primer 5'-GGGGAGCTCCTTGTCACGATTATCACAGGA$3^{\prime}$. The forward primer included a HindIII site on its $5^{\prime}$ end and the reverse primer had a $\mathrm{SacI}$ site on its $5^{\prime}$ end to facilitate cloning the amplified PCR product into the HindIII-SacI restriction enzyme sites of pNE1. An Xho1-Sac1 DNA segment was moved into the Agrobacterium tumefaciens vector pKYLX7 (Schardl et al. 1987) to complete the construction of the exon 1 hairpin (Fig. 2).

The RGH hairpin was derived by PCR from the RGH3 nucleotide sequence, one of the RGHs that was generated with the exon 2 primers and $N$. edwardsonii DNA. The reverse primer for both arms of the hairpin corresponded to the pUC18 polylinker, adjacent to the KpnI restriction enzyme site in pRGH3. The forward arm of the hairpin was derived from RGH3 nucleotide sequences 1,321 to 1,448 . It was amplified from plasmid pRGH3 using the forward primer 5'GGGCTCGAGACTACCAATCTATTCAATTG- ${ }^{\prime}$ and the reverse primer 5'-GGGGAATTCTGCGGTCGACTCTAGAGGATCC-3'. The forward primer included an XhoI site on the $5^{\prime}$ end, whereas the reverse primer had an EcoRI site on its 5' end to facilitate cloning the amplified DNA band into the XhoI-EcoRI restriction enzyme sites of the plasmid pGem$7 \mathrm{Zf}(+)$. The reverse arm of the hairpin was derived from RGH3 nucleotide sequences 1,448 to 1,047 . It was amplified from plasmid pRGH3 using the forward primer 5'-GGGGAG CTCCAAACATGGAATGCATTCTCTC- ${ }^{\prime}$ and the reverse primer 5'-GGGAAGCTTTGCGGTCGACTCTAGAGGATC C-3'. The forward primer included a SacI site on the 5' end, whereas the reverse primer had a HindIII site on its $5^{\prime}$ end to facilitate cloning into SacI-HindIII restriction enzyme sites adjacent to the forward arm. An XhoI-SacI DNA segment subsequently was moved into pKYLX7 to complete the construction of the RGH3 hairpin (Fig. 2). The nucleotide sequences of all amplified DNA segments were determined at the University of Missouri DNA Sequencing Core to verify that they matched the published $N$ gene nucleotide sequence (Whitham et al. 1994) or the RGH3 nucleotide sequence.

\section{Plant RNA extraction and cDNA synthesis.}

Detached leaves from wild-type $N$. edwardsonii and from putatively $N$-silenced $N$. edwardsonii plants were collected and each leaf was cut along the midrib into two half leaves. One half was inoculated with TMV to ensure that each leaf exhibited a delayed HR and the other half was immediately frozen at $-80^{\circ} \mathrm{C}$.

Total RNA was extracted from N. edwardsonii wild-type and the putatively $N$-silenced plants using TRIzol (Invitrogen, Carlsbad, CA, U.S.A.) according to manufacturer's protocol. Before use in RT-PCR reactions, all RNAs were treated with RNAse free DNAse at $37^{\circ} \mathrm{C}$ for $30 \mathrm{~min}$ (Promega Corp.). First-strand cDNA synthesis was carried out using the Superscript First Strand Synthesis system (Invitrogen) using oligo(dT) $)_{12-18}$ primer.

\section{Real-time PCR.}

For the $N$ gene, oligonucleotide primers were designed that amplified a 140-bp segment of exon 4 of $N$ gene. The forward primer for the $N$ gene matched nucleotide sequences 6,119 to 6,143 (TTCTTTGTACCTTTTGCTGGCTTAT) and the reverse primer matched nucleotide sequences 6,259 to 6,234 (CTCTG GTCCTTCTTTATACAACAAAC) (Levy et al. 2004). For EF$1 \alpha$, primers were selected to amplify a 143-bp product. The forward primer matched nucleotides 304 to 323 (CCTGGACA CAGGGACTTCAT) and the reverse primer matched 447 to 428 (AGCAAGCAATGCATGTTCAC) of GenBank accession D63396.

Real-time PCR was done on a DNA Engine Opticon 2 (MJ Research, Waltham, MA, U.S.A.) RT-PCR system in a total volume of $25 \mu \mathrm{l}$ per well. PCR cycles were 1 cycle of $2 \mathrm{~min}$ at $50^{\circ} \mathrm{C}$ and $15 \mathrm{~min}$ at $95^{\circ} \mathrm{C}$; followed by 45 cycles of $15 \mathrm{~s}$ at $95^{\circ} \mathrm{C}, 30 \mathrm{~s}$ at $60^{\circ} \mathrm{C}$, and $45 \mathrm{~s}$ at $72^{\circ} \mathrm{C}$; and 1 cycle of $10 \mathrm{~min}$ at $72^{\circ} \mathrm{C}$. After each PCR, a melting curve was run from 70 to $95^{\circ} \mathrm{C}$ at $0.2^{\circ} \mathrm{C}$ increments.

The mRNA levels for the genes of interest were quantified from the cycle threshold $\left(C_{t}\right)$ value, which is the PCR cycle number that generated a common signal for each gene in the exponential phase of amplification. The $C_{t}$ values were converted to template concentrations using standard curves (Giulietti et al. 2001). To correct for sampling errors, the levels of expression of each gene, as determined from their $C_{t}$ values, were normalized to the level of EF-1 $\alpha$.

\section{Evaluation of transgenic plants}

for silencing of the $\boldsymbol{N}$ family of $\boldsymbol{R}$ genes.

Detached Nicotiana leaves were lightly dusted with 600mesh Carborundum and inoculated with TMV virions at a concentration of $100 \mu \mathrm{g} / \mathrm{ml}$. Immediately after inoculation, 
the detached leaves were placed on a damp paper towel in a Ziploc bag and monitored daily for the appearance of necrotic lesions. Selected plants that responded to TMV inoculation with a delayed HR subsequently were evaluated for resistance to the Cherry strain of TBSV (Hearne et al. 1990) and CymRSV (Grieco et al. 1989). TBSV and CymRSV inocula were from infected, frozen $N$. clevelandii leaf tissue that were ground with a mortar and pestle and diluted to approximately 1:20 (wt/vol) with $50 \mathrm{mM}$ phosphate buffer (pH 7.0). The TMV-30B-GFP vector (Shivprasad et al. 1999) (kindly provided by Dr. Dennis Lewandowski) was linearized with PstI and in vitro transcription was done using a RiboMax Large-Scale RNA production system-T7 (Promega Corp.) according to the manufacturer's protocol. The Gcapped transcripts were rub inoculated onto leaves that were lightly dusted with Carborundum. GFP expression in detached leaves was visualized with a Blak-Ray long-wave ultraviolet lamp (Upland, CA, U.S.A.).

\section{ACKNOWLEDGMENTS}

This research was supported by the Missouri Agricultural Experiment Station, the Food for the 21st Century program at the University of Missouri, and the United States Department of Agriculture/National Research Initiative Competitive Grant No. 2003-35319-13778. The authors thank H. Scholthof for gifts of TBSV and CymRSV, D. Lewandowski and J. Lindbo for the gift of TMV-30B-GFP and technical assistance, K. Bilyeu for the use of the real-time PCR machine, N. Li for technical assistance in cloning RGHs, and X. Zhang for assistance with the phylogenetic analysis.

\section{LITERATURE CITED}

Altschul, S. F., Gish, W., Miller, W., Myers, E. W., and Lipman, D. J. 1990. Basic local alignment search tool. J. Mol. Biol. 215:403-410.

Baulcombe, D. C. 1999. Fast forward genetics based on virus-induced gene silencing. Curr. Opin. Plant Biol. 2:109-113.

Christie, S. R. 1969. Nicotiana hybrid developed as a host for plant viruses. Plant Dis. Rep. 53:939-941.

Chu, M., Park, J-W., and Scholthof, H. B. 1999. Separate regions on the tomato bushy stunt virus p22 protein mediate cell-to-cell movement versus elicitation of effective resistance responses. Mol. Plant-Microbe. Interact. 12:285-292.

Clamp, M., Cuff, J., Searle, S. M., and Barton, G. J. 2004. The Jalview Java alignment editor. Bioinformatics 20:426-427.

Cole, A. B., Király, L., Ross, K., and Schoelz, J. E. 2001. Uncoupling resistance from cell death in the hypersensitive response of Nicotiana species to Cauliflower mosaic virus infection. Mol. Plant-Microbe Interact. 14:31-41.

Couch, B. C., Spangler, R., Ramos, C., and May, G. 2006. Pervasive purifying selection characterizes the evolution of $I 2$ homologs. Mol. PlantMicrobe Interact. 19:288-303.

Dellaporta, S. L., Wood, J. W., and Hicks, J. B. 1983. A plant DNA minipreparation: Version II. Plant Mol. Biol. Rep. 1:19-21.

Dinesh-Kumar, S. P., and Baker, B. J. 2000. Alternatively spliced $N$ resistance gene transcripts: Their possible role in tobacco mosaic virus resistance. Proc. Natl. Acad. Sci. U.S.A. 97:1908-1913.

Felsenstein, J. 2000. PHYLIP (Phylogeny Inference Package), ed. 3.6. Distributed by the author. Department of Genetics, University of Washington, Seattle.

Flor, H. H. 1971. Current status of gene-for-gene concept. Annu. Rev. Phytopathol. 9:275-296.

Giulietti, A., Overbergh, L., Valckx, D., Decallonne, B., Bouillon, R., and Mathieu, C. 2001. An overview of real-time quantitative PCR: Applications to quantify cytokine gene expression. Methods 25:386-401.

Grieco, F., Burgyan, J., and Russo, M. 1989. The nucleotide sequence of cymbidium ringspot virus RNA. Nucleic Acids Res. 17:6383.

Hearne, P. Q., Knorr, D. A., Hillman, B. I., and Morris, T. J. 1990. The complete genome structure and synthesis of infectious RNA from clones of tomato bushy stunt virus. Virology 177:141-151.

Hehl, R., Faurie, E., Hesselbach, J., Salamini, F., Whitham, S., Baker, B., and Gebhardt, C. 1999. TMV resistance gene $N$ homologues are linked to Synchtrium endobioticum resistance in potato. Theor. Appl. Genet. 98:379-386.

Jin, H., Axtell, M. J., Dahlbeck, D., Ekwenna, O., Zhang, S., Staskawicz,
B., and Baker, B. 2002. NPK1, an MEKK1-like mitogen-activated protein kinase, regulates innate immunity and development in plants. Dev. Cell 3:291-297.

Kanazin, V., Marek, L. F., and Shoemaker, R. C. 1996. Resistance gene analogs are conserved and clustered in soybean. Proc. Natl. Acad. Sci. U.S.A. 93:11746-11750.

Kang, B., Yeam, I., and Jahn, M. M. 2005. Genetics of plant virus resistance. Annu. Rev. Phytopathol. 43:581-621.

Khetarpal, R. K., Maisonneuve, B., Maury, Y., Chalhoub, B. A., Dinant, S., Lecoq, H., and Varma, A. 1998. Breeding for resistance to plant viruses. Pages 14-32 in: Plant Virus Disease Control. A. Hadidi, R. K. Khetarpal, and H. Koganezawa, eds. American Phytopathological Society Press, St. Paul, MN, U.S.A.

Klahre, U., Crété, P., Leuenberger, S. A., Iglesias, V. A. and Meins, Jr., F. 2002. High molecular weight RNAs and small interfering RNAs induce systemic posttranscriptional gene silencing in plants. Proc. Natl. Acad. Sci. U.S.A. 99:11981-11986.

Lanfermeijer, F. C., Dijkhuis, J., Sturre, M. J. G., de Haan, P., and Hille, J. 2003. Cloning and characterization of the durable Tomato mosaic virus resistance gene Tm- $2^{2}$ from Lycopersicon esculentum. Plant Mol. Biol. 52:1039-1051.

Leister, D., Ballvora, A., and Salamini, F. 1996. A PCR-based approach for isolating pathogen resistance genes from potato with potential for wide application in plants. Nature Genet. 14:421-429.

Levy, M., Edelbaum, O., and Sela, I. 2004. Tobacco mosaic virus regulates the expression of its own resistance gene N. Plant Physiol. 135:23922397.

Liu, Y., Schiff, M., Marathe, R., and Dinesh-Kumar, S.P. 2002. Tobacco Rar1, EDS1 and NPR1/NIM1 like genes are required for $N$-mediated resistance to tobacco mosaic virus. Plant J. 30:415-429.

Liu, Y., Schiff, M., and Dinesh-Kumar, S.P. 2004. Involvement of MEK1 MAPKK, NTF6, MAPK, WRKY/MYB transcription factors, COI1 and CTR1 in $\mathrm{N}$-mediated resistance to Tobacco mosaic virus. Plant J. 38:800-809.

Lu, R., Malcuit, I., Moffett, P., Ruiz, M. T., Peart, J., Wu, A., Rathjen, J. P., Bendahmane, A, Day, L., and Baulcombe, D. 2003. High throughput virus-induced gene silencing implicates heat shock protein 90 in plant disease resistance. EMBO (Eur. Mol. Biol. Organ.) J. 22 56905699

Martin, G. B., Bogdanove, A. J., and Sessa, G. 2003. Understanding the functions of plant disease resistance proteins. Annu. Rev. Plant Biol. 54:23-61.

Matzke, M. A., Matzke, A. J. M., Pruss, G. J., and Vance, V. B. 2001. RNA-based silencing strategies in plants. Curr. Opin. Genet. Dev. 11:221-227.

Nicaise, V., German-Retana, S., Sanjuan, R., Dubrana, M. P., Mazier, M., Maisonneuve, B., Candresse, T., Caranta, C., and LeGall, O. 2003. The eukaryotic translation initiation factor $4 \mathrm{E}$ controls lettuce susceptibility to the potyvirus Lettuce mosaic virus. Plant Physiol. 132:1272-1282.

Peart, J., Cook, G., Feys, B. J., Parker J. E., and Baulcombe, D. C. 2002. An EDS1 orthologue is required for $N$-mediated resistance against tobacco mosaic virus. Plant J. 29:569-579.

Peart, J., Mestre, P., Lu, R., Malcuit, I., and Baulcombe, D. 2005. NRG1, a CC-NB-LRR protein, together with N, a TIR-NB-LRR protein, mediates resistance against Tobacco mosaic virus. Curr. Biol. 15:968-973.

Plocik, A., Layden, J., and Kesseli. R. 2004. Comparative analysis of NBS domain sequences of NBS-LRR disease resistance genes from sunflower, lettuce, and chicory. Mol. Phylogenet. Evol. 31:153-163.

Robertson, D. 2004. VIGS vectors for gene silencing: Many targets, many tools. Annu. Rev. Plant Biol. 55:495-519.

Ruffel, S., Dussault, M. H., Palloix, A., Moury, B., Bendahmane, A. Robaglia, C., and Caranta, C. 2002. A natural recessive resistance gene against Potato virus $Y$ in pepper corresponds to the eukaryotic initiation factor 4E (eIF4E). Plant J. 32:1067-1075.

Schardl, C. L., Byrd, A. D., Benzion, G., Altschuler, M. A., Hildebrand, D. F., and Hunt, A. G. 1987. Design and construction of a versatile system for the expression of foreign genes in plants. Gene 61:1-11.

Schoelz, J. E., Wiggins, B. E., Wintermantel, W. M., and Ross, K. 2006. Introgression of a Tombusvirus resistance locus from $N$. edwardsonii var. Columbia to N. clevelandii. Phytopathology 96:453-459.

Seo, Y.-S., Rojas, M. R., Lee, J.-Y., Lee, S.-W., Jeon, J.-S., Ronald, P., Lucas, W. J., and Gilbertson, R. L. 2006. A viral resistance gene from common bean functions across plant families and is up-regulated in a non-virusspecific manner. Proc. Natl. Acad. Sci. U.S.A. 103:11856-11861.

Shivprasad, S., Pogue, G. P., Lewandowski, D. J., Hidalgo, J., Donson, J., Grill, L. K., and Dawson, W. O. 1999. Heterologous sequences greatly affect foreign gene expression in tobacco mosaic virus-based vectors. Virology 255:312-323.

Smith, N. A., Singh, S. P., Wang, M.-B., Stoutjesdijk, P. A., Green, A. G., 
and Waterhouse, P. M. 2000. Total silencing by intron-spliced hairpin RNAs. Nature 407:319-320.

Soosaar, J. L. M., Burch-Smith, T. M., and Dinesh-Kumar, S. P. 2005. Mechanisms of plant resistance to viruses. Nat. Rev. Microbiol. 3:789798

Stange, C., Matus, J. T., Elorza, A., and Arce-Johnson, P. 2004. Identification and characterization of a novel tobacco mosaic virus resistance gene $\mathrm{N}$ homologue in Nicotiana tabacum plants. Funct. Plant Biol. $31: 149-158$

Thompson, J. D., Gibson, T. J., Plewniak, F., Jeanmougin, F., and Higgins, D. G. 1997. The Clustal-X Windows interface-flexible strategies for multiple sequence alignment aided by quality analysis tools. Nucleic Acid Res. 25:4876-4882.

Torres, M. A., Dangl, J. L., and Jones, J. D. 2002. Arabidopsis gp91 ${ }^{\text {phox }}$ homologues AtrbohD and AtrbohF are required for accumulation of reactive oxygen intermediates in the plant defense response. Proc. Natl. Acad. Sci. U.S.A. 99:517-522.

Vidal, S., Cabrera, H., Andersson, R. A., Fredriksson, A., and Valkonen, J. P. T. 2002. Potato gene $Y-1$ is an $N$ gene homolog that confers cell death upon infection with potato virus Y. Mol. Plant-Microbe Interact. 15:717-727.

Whitham S., Dinesh-Kumar, S. P., Choi, D., Heyl, R., Corr, C., and Baker, B. 1994. The product of the tobacco mosaic virus resistance gene $N$ : Similarity to Toll and the interleukin-1 receptor. Cell 78:1101-1115.

Yu, Y. G., Buss, G. R., and Saghai Maroof, M. A. 1996. Isolation of a superfamily of candidate disease-resistance genes in soybean based on a conserved nucleotide-binding site. Proc. Natl. Acad. Sci. U.S.A. 93:11751-11756.

Zmasek, C. M., and Eddy, S. R. 2001. ATV: Display and manipulation of annotated phylogenetic trees. Bioinformatics 17:383-384. 